\title{
Minieditorial: "Criação e Implementação de um Banco de Dados Prospectivo e Multicêntrico de Pacientes com Infarto Agudo do Miocárdio: RIAM"
}

\author{
Short Editorial: "Creation and Implementation of a Prospective Multicenter Registry of Acute Myocardial \\ Infarction: RIAM"
}

José Mariani Junior $1,2,3 \odot$

Hospital Israelita Albert Einstein, ${ }^{1}$ São Paulo, SP, Brasil

Santa Casa de São Paulo, ${ }^{2}$ São Paulo, SP, Brasil

Instituto do Coração - HCFMUSP,3 São Paulo, SP, Brasil

As doenças cardiovasculares, incluindo o infarto agudo do miocárdio (IAM), representam importante problema de saúde pública no Brasil e no mundo, com elevadas taxas de incidência e mortalidade. ${ }^{1}$ A taxa de mortalidade brasileira por esse grupo de causas $(183,3 / 100.000)^{1,2}$ encontra-se entre as maiores do mundo e é semelhante à de países como a China e do Leste Europeu. ${ }^{1,3}$

A instituição de políticas de promoção de saúde, diagnóstico precoce e tratamento efetivo estão entre as mais importantes estratégias de prevenção e tratamento dessas doenças, que ainda correspondem à principal causa de mortalidade em todo o mundo em pacientes adultos. Porém,

\section{Palavras-chave}

Doenças Cardiovasculares/mortalidade; Epidemiologia; Infarto do Miocárdio/prevenção e controle; Política de Saúde Pública; Base de Dados; Tomada de Decisões.

Correspondência: José Mariani Junior •

Hospital Israelita Albert Einstein - Medicina Intervencionista - 4 Andar -

Bloco B - Av. Albert Einstein, 627. CEP 05652-900, Morumbi, São Paulo,

SP - Brasil

E-mail: jose.mariani@einstein.br

DOI: https://doi.org/10.36660/abc.20200158 o reconhecimento bem como a mensuração real e efetiva dessas doenças ainda estão dentre as estratégias a serem otimizadas no nosso meio.

Assim, faz-se necessário conhecer e quantificar efetivamente os pacientes com diagnóstico de IAM para que medidas efetivas e assertivas possam ser tomadas na tentativa de reduzir sua morbimortalidade. Uma das maneiras mais conhecidas para esse tipo de mensuração consiste na elaboração de um banco de dados representativo e de fácil acesso e interpretação. Ainda assim, esbarramos em mais um problema, que consiste em como fazê-lo e implementá-lo. Boa parte dessas questões foram respondidas elegantemente no artigo elaborado por Vaz et al., ${ }^{4}$ intitulado: "Criação e Implementação de um Banco de Dados Prospectivo e Multicêntrico de Paciente com Infarto Agudo do Miocárdio: RIAM". ${ }^{4}$ A descrição clara e objetiva do passo a passo para a criação, implementação e expansão desse banco de dados constitui literatura rara e imperdível para todos os envolvidos na abordagem dessas patologias.

A possibilidade de termos acesso a informações geradas por bancos de dados bem desenhados e confiáveis oferece uma fotografia mais próxima da nossa realidade, aumentando a eficácia na tomada de decisões e tornando a gestão financeira mais eficiente, o que é crucial para nossas políticas de saúde pública.

\section{Referências}

1. Santos J, Meira KC, Camacho AR, Salvador PTCO, Guimaraes RM, Pierin AMG, Simoes TC, Freire FHMA. Mortalidade por infarto agudo do miocárdio no Brasil e suas regiões geográficas: análise do efeito da idade-períodocoorte. Cienc. Saúde Coletiva. 2018.23(5):1621-34.

2. Malta DC, Moura L, Prado RR, Schimidt MI, Duncan BB. Mortalidade por doenças crônicas não transmissíveis no Brasil e suas regiões, 2000 a 2011. Epidemiol Serv Saúde. 2014; 23(4):599-608.

3. Moran AE, Forouzanfar MH, Roth GA, Mensah GA, Ezzati M, Murray CJ, Naghavi M. Temporal trends in ischemic heart disease mortality in 21 world regions, 1980 to 2010: the Global Burden of Disease 2010 study. Circulation. 2014;129(14):1483-92.

4. Vaz J, Abelin AP, Schmidt MM, Oliveira PP, Gottschall CAM, Rodrigues CG, Quadros AS. Criação e Implementação de um Banco de Dados Prospectivo e Multicêntrico de Paciente com Infarto Agudo do Miocárdio: RIAM. Arq Bras Cardiol. 2020; 114(3):446-455. 